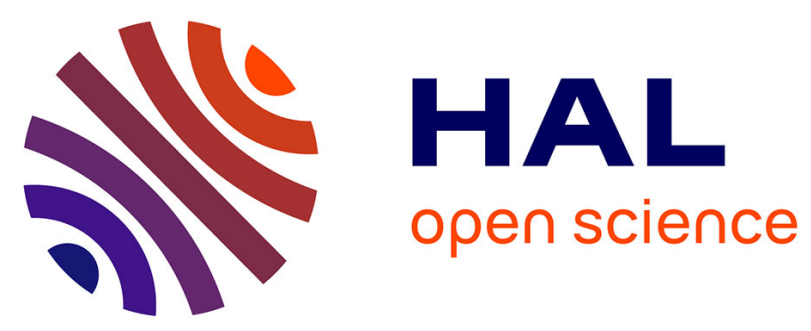

\title{
Evidence for cooperative mineralization of diuron by Arthrobacter sp. BS2 and Achromobacter sp. SP1 isolated from a mixed culture enriched from diuron exposed environments
}

\author{
M. Devers Lamrani, S. Pesce, N. Rouard, F. Martin Laurent
}

\section{To cite this version:}

M. Devers Lamrani, S. Pesce, N. Rouard, F. Martin Laurent. Evidence for cooperative mineralization of diuron by Arthrobacter sp. BS2 and Achromobacter sp. SP1 isolated from a mixed culture enriched from diuron exposed environments. Chemosphere, 2014, 117, pp.208-215. 10.1016/j.chemosphere.2014.06.080 . hal-01130203

\author{
HAL Id: hal-01130203 \\ https://hal.science/hal-01130203
}

Submitted on 11 Mar 2015

HAL is a multi-disciplinary open access archive for the deposit and dissemination of scientific research documents, whether they are published or not. The documents may come from teaching and research institutions in France or abroad, or from public or private research centers.
L'archive ouverte pluridisciplinaire HAL, est destinée au dépôt et à la diffusion de documents scientifiques de niveau recherche, publiés ou non, émanant des établissements d'enseignement et de recherche français ou étrangers, des laboratoires publics ou privés. 


\title{
Evidence for cooperative mineralization of diuron by Arthrobacter sp. BS2 and Achromobacter sp. SP1 isolated from a mixed culture enriched from diuron exposed environments
}

\author{
Marion Devers-Lamrani ${ }^{\mathrm{a}}$, Stéphane Pesce ${ }^{\mathrm{b}}$, Nadine Rouard $^{\mathrm{a}}$, Fabrice Martin-Laurent ${ }^{\mathrm{a}}$ \\ a INRA, UMR 1347 Agroécologie, 17 rue Sully, BP 86510, 21065 Dijon Cedex, France \\ b Irstea, Centre de Lyon-Villeurbanne, UR MALY, 5 rue de la Doua, CS 70077, 69626 Villeurbanne Cedex, \\ France
}

\section{Highlights}

- Isolation of Arthrobacter diuron-degrading strains from soils and sediments.

- Achromobacter 3,4-DCA degrading strain isolation from sediments.

- Arthrobacter strain transformed diuron to 3,4-DCA with PuhA enzyme.

- Achromobacter isolate transformed 3,4-DCA with DcaQ enzyme.

- Mixed culture of Arthrobacter and Achromobacter mineralized 14C-diuron to 14CO2.

\begin{abstract}
Diuron was found to be mineralized in buffer strip soil (BS) and in the sediments (SED) of the Morcille river in the Beaujolais vineyard repeatedly treated with this herbicide. Enrichment cultures from BS and SED samples led to the isolation of three bacterial strains transforming diuron to 3,4dichloroaniline (3,4-DCA) its aniline derivative. 16S rRNA sequencing revealed that they belonged to the genus Arthrobacter (99\% of similarity to Arthrobacter globiformis strain K01-01) and were designated as Arthrobacter sp. BS1, BS2 and SED1. Diuron-degrading potential characterized by sequencing of the puhA gene, characterizing the diuron-degradaing potential, revealed $99 \%$ similarity to A. globiformis strain D47 puhA gene isolated a decade ago in the UK. These isolates were also able to use chlorotoluron for their growth. Although able to degrade linuron and monolinuron to related aniline derivatives they were not growing on them. Enrichment cultures led to the isolation of a strain from the sediments entirely degrading 3,4-DCA. 16S rRNA sequence analysis showed that it was affiliated to the genus Achromobacter (99\% of similarity to Achromobacter sp. CH1) and was designated as Achromobacter sp. SP1. The dcaQ gene encoding enzyme responsible for the transformation of 3,4-DCA to chlorocatechol was found in SP1 with 99\% similarity to that of Comamonas testosteroni WDL7. This isolate also used for its growth a range of anilines (3-chloro-4methyl-aniline, 4-isopropylaniline, 4-chloroaniline, 3-chloroaniline, 4-bromoaniline). The mixed culture composed of BS2 and SP1 strains entirely mineralizes 14C-diuron to 14CO2. Diuronmineralization observed in the enrichment culture could result from the metabolic cooperation between these two populations.
\end{abstract}




\section{Keywords}

Pesticide; Biodegradation; Soil microbiology; Buffer strip; Sediments; 3,4-DCA

\section{Introduction}

The diuron (DIU, 3-(3,4-dichlorophenyl)-1,1-dimethylurea) is widely used on a range of crops to control the development of weeds. It is also used in urban areas, as an algicide in antifouling paints and as herbicides on non-crop areas. Having half live ranging between 100 and $134 \mathrm{~d}$ (Moncada, 2005), DIU is relatively persistent in soil. Although considered to be moderately mobile, it contaminates surface- and ground-water resources world widely (Schuler and Rand, 2008 and Stork et al., 2008). Environmentally realistic concentrations of DIU can affect aquatic microalgal (Pesce et al., 2011) and bacterial communities (Tadonleke et al., 2009). Combined presence of DIU and its main metabolites $\mathrm{N}$-(3,4-dichlorophenyl)-N-methylurea and 3,4-dichloraniline (3,4-DCA) has been reported in surface freshwaters (Claver et al., 2006 and Morin et al., 2009), this last one being highly toxic to the bacteria Vibrio fischeri and the Ciliate Tetrahymena pyriformis ( Bonnet et al., 2007) but also to macro-organisms, with no observed effect concentrations of only a few $\mu \mathrm{g} \mathrm{L-1}$ ( Giacomazzi and Cochet, 2004). DIU has been classified as a primary dangerous substance (Directive 2000/60/CE) and accordingly it has been banned in Europe but remains used in numerous regions of the world.

The major route for natural attenuation of DIU in the environment is microbial degradation (Sharma and Suri, 2011). Several studies showed its rapid degradation in different soils repeatedly treated with DIU in reason of the emergence of bacterial populations using it as a nutrient and an energy source (Dellamatrice and Monteiro, 2004 and Pesce et al., 2009). Various microorganisms such as Arthrobacter globiformis D47 ( Cullington and Walker, 1999) able to transform DIU have been isolated from various contaminated environments ( Sorensen and Aamand, 2003). Except for Variovorax sp. SRS16 which entirely mineralizes DIU ( Sorensen et al., 2008), most of the isolates transform DIU to 3,4-DCA which accumulates in soil. Strains isolated from DIU-contaminated soils, Pseudomonas fluorescens 26-K and Acinetobacter baylyi strain GFJ2 ( Travkin et al., 2003 and Hongsawat and Vangnai, 2011) have been shown to degrade 3,4-DCA. The importance of mixed bacterial cultures for the complete mineralization of DIU was underlined and catabolic cooperation between microbial populations to fulfill DIU mineralization was hypothesized ( Sorensen et al., 2008 and Ellegaard-Jensen et al., 2014). Only two genes coding for the first enzyme involved in the transformation of dimethyl-substituted phenylureas into their aniline derivatives have been reported; the phenylurea hydrolase A gene (puhA) was the first one identified in A. globiformis strain D47 ( Turnbull et al., 2001) and the phenylurea hydrolase B gene (puhB) was characterized in Mycobacterium brisbanense JK1 isolated from an Australian soil ( Khurana et al., 2009). These two genes encode amidohydrolases sharing $82 \%$ identity at the amino acid level but diverged from other related sequences forming a amidohydrolase superfamily sub-group ( Khurana et al., 2009). Recently, dcaQTA1A2BR genes encoding a multi-component chloroaniline dioxygenase responsible for 3,4DCA degradation were identified in Variovorax sp. SR16 ( Bers et al., 2011).

Studies carried out at a small-scale watershed level (Morcille river, Beaujolais, France) constituted of a vineyard treated with DIU, a grass buffer strip adjacent to it and the sediments of this river have clearly shown that in situ DIU exposure provokes an increase in the tolerance of phototrophic biofilm communities towards DIU ( Pesce et al., 2010a) and the DIU degrading potential of both terrestrial and aquatic microbial communities ( Pesce et al., 2009). DIU-degrading genetic potential quantified by quantitative PCR targeting puhA and puhB genes was detected in both terrestrial and aquatic compartments showing DIU-degrading abilities ( Pesce et al., 2013). At the watershed level, soil erosion was suggested as a possible mechanism responsible of the transfer of the DIU genetic potential from the soil to the sediments ( Pesce et al., 2010b). 
Even though bacterial populations, genes and catabolic enzymes involved in DIU transformation have been identified, not much is known about the functioning of the DIU-degrading microbial community. In order to fill this gap of knowledge, we carried out experiments aiming at isolating and characterizing members of DIU-degrading microbial communities enriched from soil and sediment samples collected from the Morcille watershed. Isolated members able to degrade DIU or its aniline derivative 3,4-DCA were taxonomically characterized by sequencing 16S rRNA gene. Degrading genetic potential was monitored by studying puhA and puhB or dcAQ genes involved in DIU or 3,4DCA transformation, respectively. The ability of these bacterial strains to degrade various phenylureas and anilines was studied. The efficiency of mixed culture made of DIU- and 3,4-DCA degraders for DIU mineralization was estimated.

\section{Material and Methods}

\subsection{Soil and sediments}

Soil was collected from upper horizon $(0-20 \mathrm{~cm})$ of a grass buffer strip (BS) located (longitude $4^{\circ} 60^{\prime} \mathrm{E}$, latitude $46^{\circ} 15^{\prime} \mathrm{N}$ ) at the interface between a vineyard and the Morcille river, a small first-order stream (7 km long) subjected to a strong agricultural pressure (Beaujolais, France). A composite BS soil sample was prepared according to ISO 10381-6 guidelines. Sediment samples (SED) were collected in the downstream section of the Morcille river (Saint Ennemond station). Soil and sediments were sieved to $5 \mathrm{~mm}$ and stored at $4{ }^{\circ} \mathrm{C}$ until their use.

\subsection{Enrichment procedure}

Enrichment cultures were done starting from either BS or SED samples. About $10 \mathrm{~g}$ of soil or sediment equivalent dry weight was suspended in $90 \mathrm{~mL}$ of mineral salt (MS) medium (Devers et al., 2005) added with sodium citrate (1 g L-1) and DIU (MS-DIU, $25 \mathrm{mg} \mathrm{L}-1)$ as sole nitrogen source, incubated at $20^{\circ} \mathrm{C}$ under agitation $(150 \mathrm{rpm})$. Aliquots were taken regularly and DIU remaining in the medium was quantified using HPLC. When about $50 \%$ of the initial amount was dissipated, $10 \mathrm{~mL}$ of this suspension was transferred to $90 \mathrm{~mL}$ of fresh MS-DIU medium and incubated under the same conditions. After 3 enrichment cycles, $100 \mu \mathrm{L}$ serial tenfold dilutions (from 10-3 to 10-6) were plated onto solid MS-DIU medium or MS-3,4-DCA medium (1 g L-1). Single colonies isolated from the plates were stricken on new plates to purify isolates. Pure colonies were selected for DIU or 3,4-DCA degradation in corresponding liquid media incubated at $28{ }^{\circ} \mathrm{C}$ under agitation $(150 \mathrm{rpm})$. After a few days, the bacterial cells were harvested (3,220 g, $10 \mathrm{~min})$ and the supernatant was analyzed by HPLC to determine the amount of the remaining DIU or 3,4-DCA.

\subsection{DNA extraction}

DNA was extracted from $75 \mathrm{~mL}$ of bacterial suspension using the QIAGEN Blood and Cell culture DNA Midi Kit (QIAGEN, France).

\subsection{PCR analysis}

PCR were conducted in $25 \mu \mathrm{L}$ by using $25 \mathrm{ng}$ of extracted DNA, $0.2 \mu \mathrm{M}$ of dNTPs, $1 \mu \mathrm{M}$ of specific primers (puhA/puhB, dcaQ and 16S rRNA primers according to Pesce et al. (2013), Bers et al. (2011) and Gürtler and Stanisich (1996)), and 1.25 U of Taq DNA polymerase (Q-biogene, France). DNA amplifications were carried out in a PTC 200 gradient cycler (MJ Research, Waltham, MA, USA) as follows: $5 \mathrm{~min}$ at $95{ }^{\circ} \mathrm{C}, 35$ cycles of $1 \mathrm{~min}$ at $94{ }^{\circ} \mathrm{C}, 1 \mathrm{~min}$ at annealing temperature and $2 \mathrm{~min}$ at 72 ${ }^{\circ} \mathrm{C}$, plus an additional 10 -min cycle at $72{ }^{\circ} \mathrm{C}$. PCR products were separated by electrophoresis and visualized under UV.

\subsection{Repetitive extragenic palendromic (REP)-PCR}

REP PCR was carried out in a $25-\mu \mathrm{L}$ mixture using $50 \mathrm{ng}$ of bacterial DNA as template and separated by electrophoresis according to Devers et al. (2005). 


\subsection{Southern blot analysis}

NruI or SmaI digested DNA was separated by electrophoresis, in the presence of digoxigenin-labelled DNA molecular weight marker II (Roche Applied Science, France) and vaccum-transferred to a Biodyne Plus membrane (Gelman Sciences, Merck Eurolab, France). The puhA and dcaQ probes labelled with digoxigenin produced by PCR were hybridized as previously described ( Devers et al., 2005).

\subsection{DNA sequencing}

16S rRNA PCR products were cloned into the pGemT-EasyII vector (Promega, Madison, WI, USA). Recombinant clones were identified by a SP6/T7 PCR and plasmids extracted using QIAprep spin miniprep kit and sequenced by Beckman Coulter Genomics (Takeley, USA). The puhA gene, its upstream (For_CCGTAGAGCTCGAAGAGGAA; Rev_GCATCGGTGTTCATGAGGAT) and downstream (For_ GAGGTCATGTTCGAGGAAGC; Rev_TCGGAGGTGTAGCGGTAGAG) sequences, and the partial sequence of dcaQ gene were directly sequenced from PCR products (GATC Biotech, France). Sequences were assembled using SeqMan software (DNASTAR) Sequence comparison was done using BlastN (http://www.ncbi.nlm.nih.gov/BLAST). Multiple alignments were done using ClustalX ( Thompson et al., 1997). Phylogenetic tree was processed from the alignment with the neighbor joining method using NJ Plot.

The 16S rRNA sequences of BS1, BS2, and SED1 DIU-degrading strains and of SP1 3,4-DCA degrading strain were deposited into the GenBank database (KJ162357, KJ127007, KJ162358, KJ127008, respectively). puhA, puhB and dcaQ sequences, were deposited in the GenBank database (KJ127011, KJ127010, KJ127009, respectively).

\subsection{Mineralization of diuron}

BS2 and SP1 strains were grown in MS-DIU or MS-3,4-DCA media at $28^{\circ} \mathrm{C}$, and then centrifuged at $6000 \mathrm{rpm}$ for $5 \mathrm{~min}$. The pellets were washed twice in Knapp buffer (Devers et al., 2005) and resuspended to an OD (600 nm) of 0.2 in Knapp buffer containing $30 \mathrm{mg} \mathrm{L}-1$ of DIU and $170 \mathrm{~Bq}$ $\mathrm{mL}-1$ of $14 \mathrm{C}$ ring-labelled DIU. Mixed bacterial culture suspensions were incubated at $28{ }^{\circ} \mathrm{C}$ in a sterile jar under agitation $(150 \mathrm{rpm}) .14 \mathrm{CO} 2$ was trapped in $5 \mathrm{~mL}$ of $0.2 \mathrm{M} \mathrm{NaOH}$ and radioactivity was periodically measured by liquid scintillation counting (Packard). This experiment was performed in triplicate.

The DIU-mineralization parameters were determined using the Gompertz model (Gompertz, 1825) with the SigmaPlot 4.0 software: $\mathrm{y}=\mathrm{a} \exp (-\exp (-\mathrm{k}(\mathrm{t}-\mathrm{ti})))$, where $\mathrm{y}$ is the percentage of DIU mineralization (\%), a the maximum percentage of DIU mineralization (\%); $\mathrm{k}$ the maximum rate of DIU mineralization ( $d-1)$; $t$ the time $(d)$, and ti the abscissa of the inflection point $(d)(p<0.005, n=3$, Student $t$ test).

\subsection{Diuron and 3,4-DCA degradation}

DIU or 3,4-DCA degradation ability was evaluated. To produce microbial biomass, cell aliquots were inoculated in MS-DIU or MS-3,4-DCA medium and incubated at $28{ }^{\circ} \mathrm{C}$ at $150 \mathrm{rpm}$. The cells were centrifuged at $6000 \mathrm{rpm}$ for $5 \mathrm{~min}$, and the pellets were washed twice in Knapp buffer. The cells were then suspended at an OD600 of 0.2 in Knapp buffer containing either DIU or 3,4-DCA (30 mg L-1), and incubated as previously described. At different times of incubation, supernatant was collected and analyzed by HPLC (LC Star system, Varian) equipped with a Microsorb-MV C18 column (length 25 $\mathrm{cm}$, internal diameter $4.6 \mathrm{~mm}$, Varian). The solvent system used was methanol added with $0.1 \%$ of H3PO4 (75/25; v/v) delivered at a flow rate of $1 \mathrm{~mL}$ min-1. DIU and 3,4-DCA were detected at 254 $\mathrm{nm}$ at different retention times. These experiments were performed in triplicate and a control was included. Kinetics parameters were determined using the Gompertz model as described in Section 2.8. 


\subsection{Characterization of degrading- or growing-capabilities of the isolated strains}

DIU- or 3,4-DCA degrading bacterial strains were grown in corresponding MS medium. Degrading capabilities were assessed by resting cell experiments: bacterial cultures were washed twice in Knapp buffer and then suspended at 0.2 OD600nm in Knapp buffer containing $50 \mathrm{mg} \mathrm{L}-1$ of the phenylureas (isoproturon, linuron, monolinuron, chlorotoluron) or anilines (4- isopropyl-aniline, 3,4-DCA, 3chloroaniline, 4-chloroaniline, 4-bromoaniline and 3-chloro-4-methylaniline). They were incubated at $28{ }^{\circ} \mathrm{C}$ under agitation. Aliquots were regularly taken, centrifuged $(3,220 \mathrm{~g}, 10 \mathrm{~min})$ and the supernatants were analyzed by HPLC. Three replicates were performed for each compound.

Growing capabilities were assessed by inoculating hardly any of bacterial culture $(0.002$ OD600nm) in corresponding MS-medium added with sodium citrate $(1 \mathrm{~g} \mathrm{~L}-1)$ and each of the phenylureas or in MS-medium with each of the anilines.

\section{Results}

\subsection{Isolation of diuron-degrading strains}

In a previous study, Pesce et al. (2009) showed that repeated applications of DIU had led to the adaptation of the microflora to the mineralization of DIU in the vineyard soil, in the adjacent BS and in the sediments (SED) of the receiving river. To isolate the microorganisms responsible for this mineralization, enrichments were performed on BS and SED samples leading to the isolation of consortia able to degrade DIU without accumulation of metabolites. From these consortia, trials were conducted to isolate pure bacterial strains involved in the process of DIU degradation.

As a result, we isolated three bacteria able to grow on MS-medium containing DIU as sole nitrogen source namely BS1, BS2, and SED1 originating from BS- and SED- samples, respectively. HPLC analyses showed that DIU was entirely dissipated during the growth of these isolates and that concomitantly a metabolite presenting the same retention time that 3,4-DCA was accumulated (data not shown).

To taxonomically characterize these three DIU-degrading isolates, their 16S rRNA sequences were analysed revealing that they were taxonomically close to A. globiformis strain K01-01 $(99.3 \%$ of similarity) (Figs. 1 and SM1 of Supplementary Material). Isolates BS1, BS2 and SED1 were named Arthrobacter sp. BS1, Arthrobacter sp. BS2 and Arthrobacter sp. SED1, respectively. They were classified as Bacteria, phylum Actinobacteria, order Actinomycetales, family Micrococcaceae and genus Arthrobacter. These three strains presented identical REP-PCR patterns ( Fig. 2) suggesting that they are either identical or closely related deriving from the same ancestor.

Strain BS2 was further used to characterize DIU-degrading ability in Knapp buffer containing $30 \mathrm{mg}$ $\mathrm{L}-1$ of DIU $(128 \mu \mathrm{M})$. HPLC monitoring showed that DIU was entirely degraded within $24 \mathrm{~h}$, the maximum degradation rate being of $24.8 \pm 1.9 \mu \mathrm{M}$ h-1 (Fig. 3, panel A). Concomitantly, the accumulation of a metabolite showing identical retention time to that of 3,4-DCA was observed. Its accumulation was equimolar to the dissipation of DIU, its maximum apparition rate being of $24.5 \pm$ $3.5 \mu \mathrm{M} \mathrm{h}-1$. The ability of strain BS2 to degrade and to grow on different phenylureas was checked (Table 1) showing that it was able to use DIU and chlorotoluron for its growth. Interestingly, even if resting cells showed that BS2 was able to degrade linuron and monolinuron to their corresponding aniline derivatives, it cannot grow on them. Finally, BS2 was unable to degrade isoproturon and to use it for its growth (Table 1). 

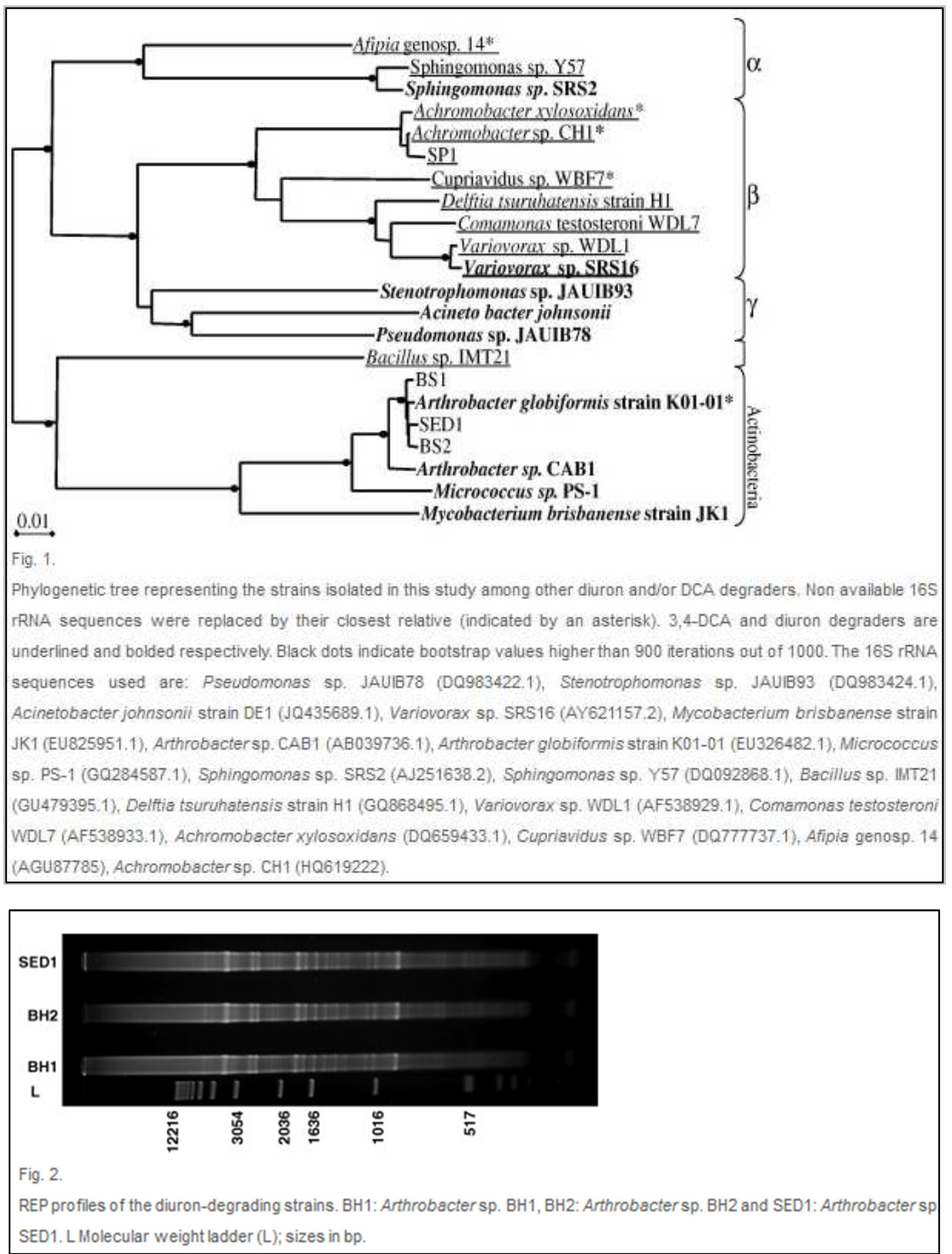

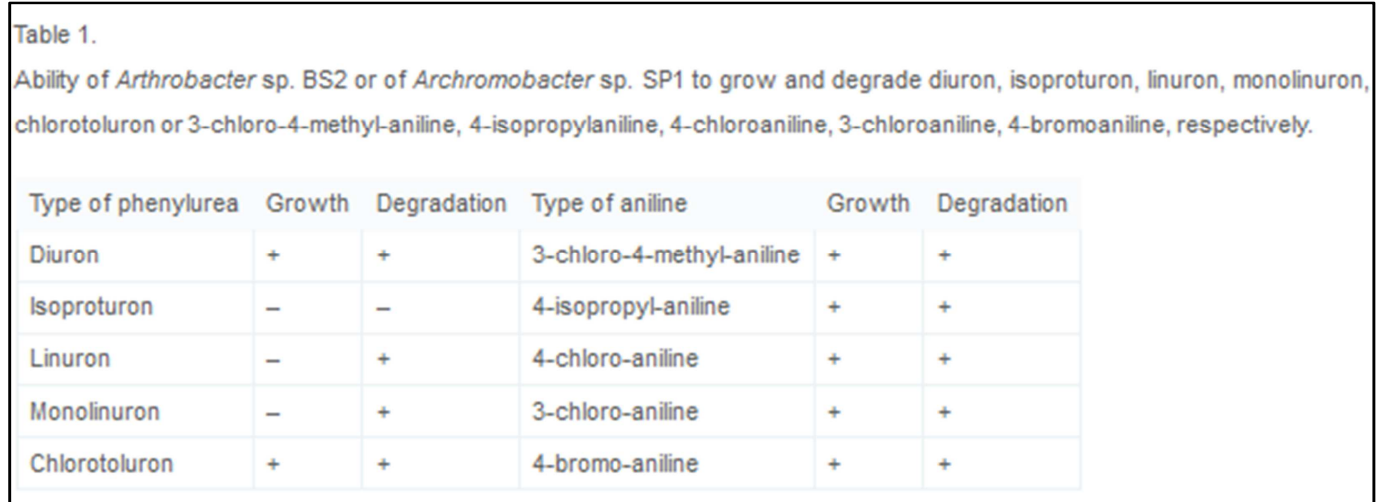



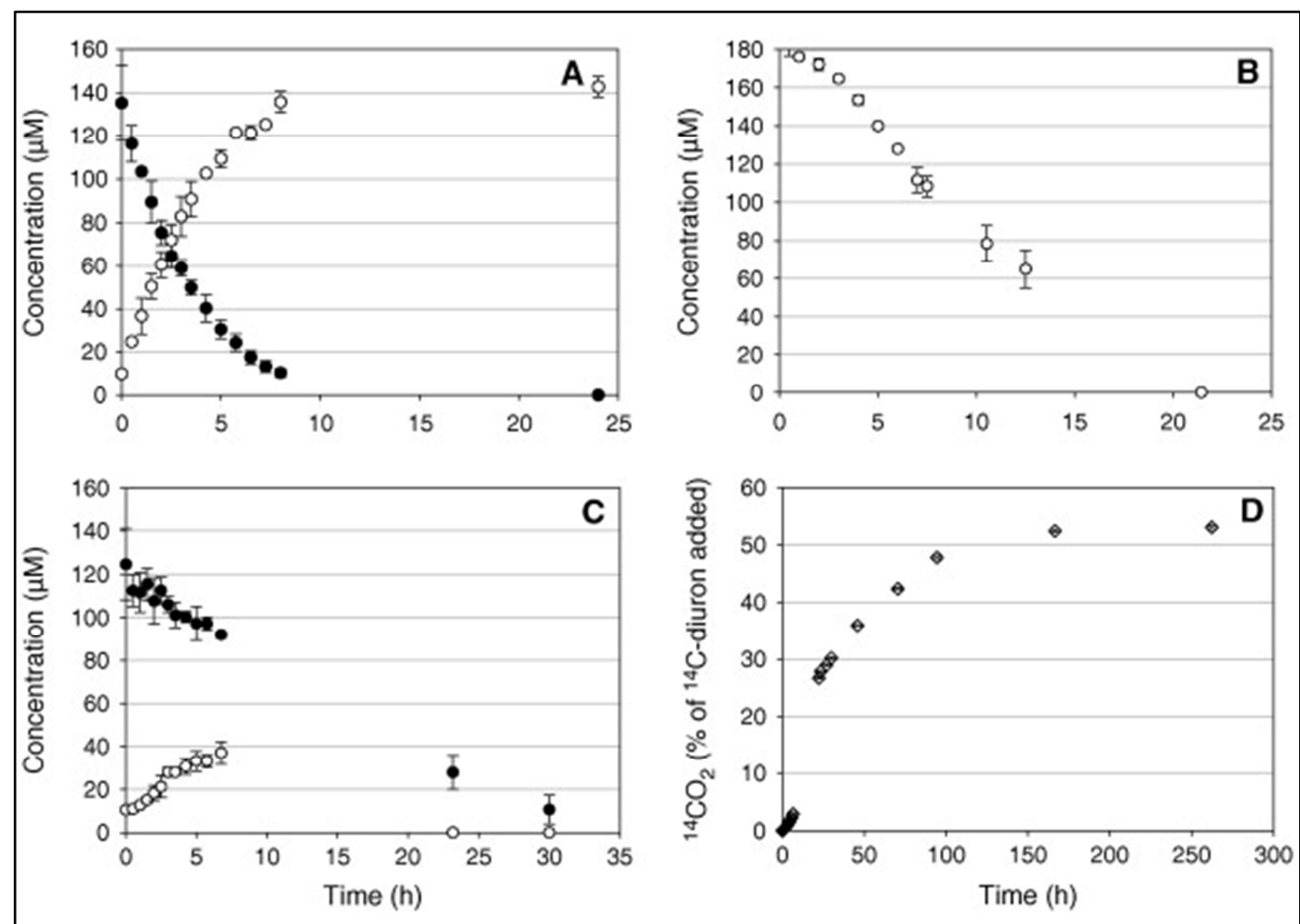

Fig. 3 .

Panel A. Degradation of diuron by Arthrobacter sp. BH2. Kinetics of diuron dissipation (full circle) and 3,4-DCA accumulation (open circle) are shown. Panel B. Degradation of 3,4-DCA by Achromobacter sp. SP1. Panel C. Degradation of diuron by a consortium made of both strains. Kinetics of diuron dissipation (full circle) and 3,4-DCA transitory accumulation (open circle) are shown. PanelD. mineralization of diuron by the consortium composed of both strains. Standard deviations are indicated $(n=3)$.

puhA and puhB DIU-degrading gene sequences, were searched by PCR on DNA extracted from the enrichment culture or pure degrading-strains. puhB amplicon of $665 \mathrm{bp}$ was obtained with DNA from enrichment cultures. Interestingly, PCR analyses carried out with the pure degrading strains produced puhA amplicon and not puhB (data not shown). Sequencing of the PCR products revealed that the puhB amplicon amplified from the enrichment culture shared more than $95 \%$ of similarity with the puhB gene from M. brisbanense strain JK1 (593/624 bp). In addition, sequencing of puhA amplicons amplified from DIU-degrading strains were $99 \%$ of similarity with that of A. globiformis strain D47 $(653 / 655 \mathrm{pb})$. Southern blot analysis of DIU-degrading isolates hybridized with puhA probe gave a single signal on a $3.1 \mathrm{~kb}$ NruI fragment and $8.2 \mathrm{~kb}$ SmaI fragment ( Fig. 4).

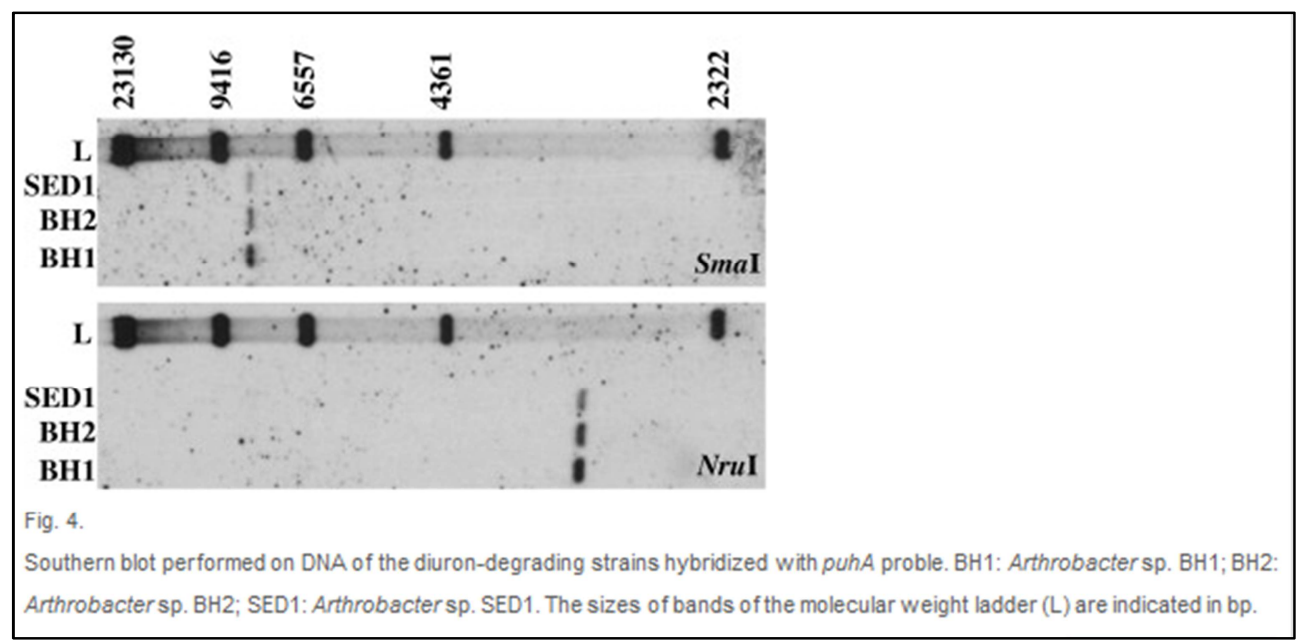


PCR reactions were performed to determine whether the upstream- and downstream-sequences surrounding puhA gene in BS2 strain were similar to those of A. globiformis strain D47 (EU851877). Amplifications performed using primers either located 464 bp upstream or 524 bp downstream puhA coupled with primers anchored in puhA sequence yielded in two PCR products of expected sizes (data not shown). Sequencing of these PCR products led to the complete sequence of puhA as well as its upstream (444 bp) and downstream (473 pb) sequence on BS2 (GenBank accession number KJ127011). It revealed that puhA sequence shared $99.8 \%$ similarity with puhA form A. globiformis strain D47 (1369/1371 bp). At the protein level, two amino acids were substituted as compared to the known PuhA (146L-146M and 335L-335I), these substitutions being positive. We found that both the sequences located upstream and downstream to puhA were $100 \%$ similar to those found in A. globiformis strain D47 ( Fig. 5).

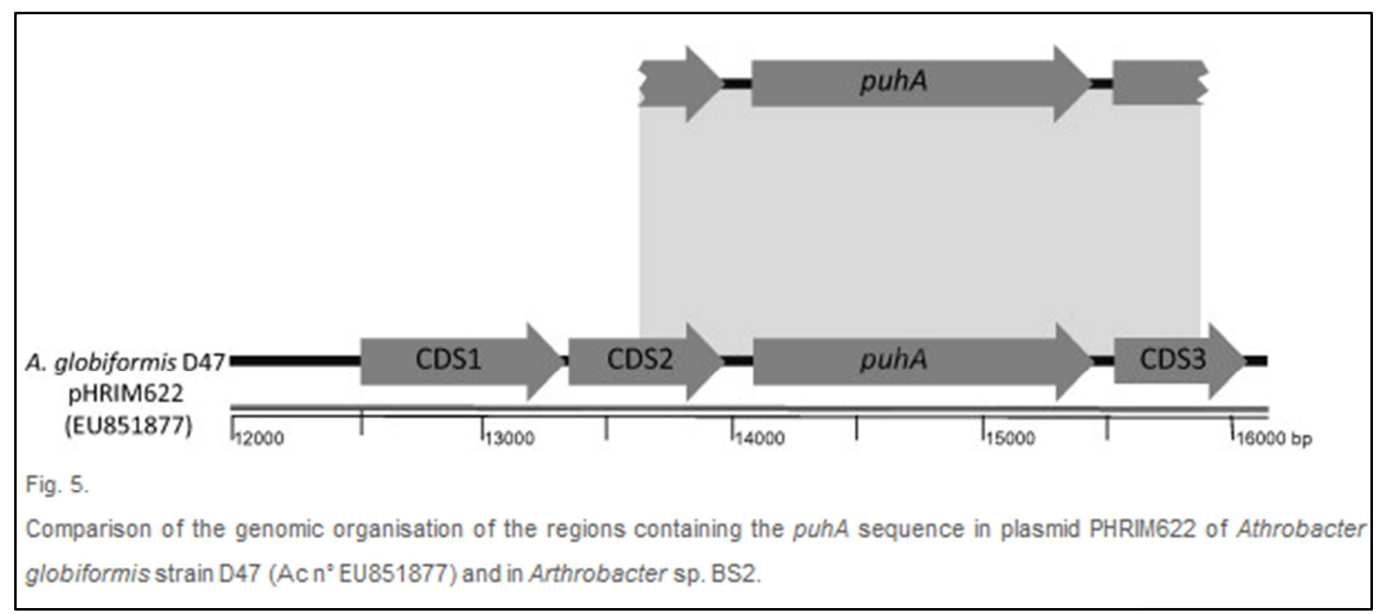

\subsection{Isolation of 3,4-DCA degraders}

We attempted to isolate the strains responsible for the degradation of 3,4-DCA in the enrichment culture. One isolate able to degrade 3,4-DCA was isolated from the SED enrichment culture using MS medium containing 3,4-DCA as sole nitrogen and carbon sources. Unfortunately, isolation of 3,4-DCA degraders from BS enrichment culture was unsuccessful. 16S rRNA sequence indicated that this was highly similar to Achromobacter sp. Ch1 (99.4\% on 1453 bp) ( Fig. 1). Consequently, the isolate was named Achromobacter sp. SP1 and classified as Bacteria, class Betaproteobacteria, order Burkholderiales, family Alcaligenaceae and genus Achromobacter.

The ability of SP1 to degrade 3,4-DCA was evaluated by monitoring its dissipation by HPLC (Fig. 3, panel B). SP1 was able to completely dissipate $185 \mu \mathrm{M}$ of 3,4-DCA in $21 \mathrm{~h}$, and no metabolites were detected. The study of catabolic ability of SP1 was assessed against a range of anilines showed that it was able to grow and degrade 3-chloro-4-methyl-aniline, 4-isopropylaniline, 4-chloroaniline, 3chloroaniline and 4-bromoaniline (Table 1). PCR targeting the gene dcaQ encoding a part of a multicomponent enzyme responsible for 3,4-DCA degradation was performed on SP1. It revealed the presence of this gene sharing 99\% similarity with the one from Comamonas testosteroni (227/229 bp); at the amino acid level, it shares 93-97\% identity with other known DcaQ sequences.

\subsection{Diuron mineralizing ability of the Arthrobacter sp. BS2 and Achromobacter sp. SP1 consortium}

The ability of an artificial consortium made of BS2 and SP1 to entirely degrade DIU was assessed revealing that it was able to entirely dissipate DIU within $35 \mathrm{~h}$. Transient accumulation of 3,4-DCA was observed (Fig. 3, panel C). This accumulation was equimolar to the degradation of DIU indicating that in the early stage, SP1 was not active. However, after 20 d incubation, no more 3,4-DCA was detected in the culture medium indicating that SP1 had degraded it all. DIU mineralization observed in the artificial consortium showed that more than $50 \%$ of $14 \mathrm{C}$-DIU evolved to $\mathrm{CO} 2$ within $5 \mathrm{~d}$ of incubation (Fig. 3, panel D). 


\section{Discussion}

DIU mineralization has already been reported from different compartments at the watershed level of the Morcille river (Pesce et al., 2009). Our study aimed at studying microbial populations involved in DIU degradation. To do so samples were collected in the buffer strip (BS) and sediments (SED) of the watershed. As expected BS and SED samples periodically exposed to DIU, were able to mineralize up to $20 \%$ of the $14 \mathrm{C}$-ring-labeled DIU. Analyse of the DIU-genetic potential indicated the presence of puhB gene sequence in enrichments cultures confirming Pesce et al. (2013) reporting its presence in a vineyard plot adjacent to BS. Surprisingly, puhA sequence which was neither detected in the enrichment culture nor in BS and SED samples was found in DIU-degrading isolates. To explain this discrepancy between molecular and Pasteurian approaches, one could hypothesize on one hand, that puhA sequences were below PCR detection limit in the enrichment cultures or on the other hand, that microbial populations harbouring puhB were not culturable in our conditions. Anyhow, this is the first report of the co-occurrence of puhA and puhB genes in the same environment. It confirmed the existence of DIU-degrading genetic potential and of DIU-mineralizing ability among BS and SED microflora which might be attributed to the adaptation of indigenous microbiota to metabolize DIU.

Enrichment cultures were conducted from composite BS or SED samples with DIU used as a sole nitrogen source. Although both BS and SED enrichment cultures were able to mineralize DIU, no bacterial populations able to fully mineralize this herbicide was isolated. Isolation trials led to the isolation of three DIU-degrading pure isolates, two originating from BS (i.e. BS1 and BS2) and one from SED samples (SED1). This further confirms that both BS and SED samples harbour similar microbial populations able to transform DIU as observed earlier (Pesce et al., 2009 and Pesce et al., 2010b). None of these isolates mineralize DIU, but equimolarly transformed DIU to 3,4-DCA. These isolates did not only have similar phenotypes and REP profiles. In addition, their 16S rRNA sequences were up to $99.3 \%$ similar to A. globiformis strain K01-01. Interestingly, 16S rRNA sequences showed 97\% with that of A. oxydans (X83408) the closest relative of A. globiformis strain D47 and 99\% of similarity with A. globiformis (X80736) ( Turnbull et al., 2001). Although Arthrobacter sp. BS1, BS2 and SED1 are grouped with A. globiformis strain D47 within the A. globiformis group, our isolates diverged slightly from the last which is inferred to A. oxydans type. The isolation of BS1, BS2 and SED 1 isolates from Beaujolais vineyard (France) highly similar to A. globiformis D47 isolated one decade ago from Deep Slade soil (UK) poses a challenging question regarding their evolutionary origin ( Cullington and Walker, 1999). It suggests that DIU-degradation ability is specific to Arthrobacter taxa. One may nevertheless keep in mind that this specificity can also reflect a good culturability of Arthrobacter species compared to possible other DIU-degrading populations. However, similar observations have been done for other phenylureas such as isoproturon which was found to be mineralized by different populations related to Sphingomonads such as Sphingomonas sp. SRS2, Sphingobium YBL10 and Sphingomonas sp. SH isolated from Danish, Chinese and French agricultural soils, respectively ( Sorensen et al., 2001, Sun et al., 2009 and Hussain et al., 2011). However, the opposite figure is found for s-triazine degraders which are spread in various Gramnegative and Gram-positive bacteria populations ( Udikovic-Kolic et al., 2012). In order to study in more details, DIU-degrading genetic potential, we searched among our isolates for the presence of phenylurea hydrolase genes. Although puhB gene was detected in the enrichment cultures, only puhA was found in our isolates, indicating that we only partially characterized the populations involved in the biodegradation of DIU. Interestingly, Southern blot analysis revealed the existence of $8.2 \mathrm{~kb}$ SmaI fragment harbouring puhA gene in our isolates corresponding to a fragment similar in size of pHRIM622 the catabolic plasmid of A. globiformis strain D47 ( Khurana et al., 2009). In addition, sequencing showed that the puhA sequence of BS2 was $99.8 \%$ to that of A. globiformis strain D47 and that upstream and downstream sequences of BS2 were $100 \%$ similar to that of A. globiformis strain D47. This indicates that the DIU-degrading genetic potential of our three isolates is similar to that of A. globiformis strain D47 although they have been isolated from distinct geographic regions at different periods of time. Interestingly, one could noticed that BS1/BS2 and SED1strains isolated from BS and SED samples respectively, were belonging to the same genus, having identical REP profiles 
and DIU-degrading genetic potential thereby suggesting that similar DIU-degraders can be found in different environmental niches. This is in accordance with previous report highlighting the importance of run-off in the connection of terrestrial and aquatic compartments ( Pesce et al., 2010b).

We showed that like A. globiformis strain D47 ( Cullington and Walker, 1999), BS2 was able to transform a range of phenylureas including DIU, chlorotoluron, linuron and monolinuron to corresponding derivative anilines but not isoproturon. This might be explained by the catalytic efficiency of PuhA which is higher for DIU or chlorotoluron ( $\mathrm{kcat} / \mathrm{Km}$ value of 0.5 and $0.2 \mu \mathrm{M}-1$ min-1, respectively) than for isoproturon (kcat/Km value of $0.0074 \mu \mathrm{M}-1 \mathrm{~min}-1$ ) ( Khurana et al., 2009). Interestingly, one could observe that BS2 only grew on N-Dimethyl ureas (DIU and chlorotoluron) and not on N-Methoxy-N-methyl ureas (linuron and monolinuron) although it was able to degrade all of them. One could hypothesize either that DIU degradation pathway is induced by NDimethyl ureas but not by N-Methoxy-N-methyl ureas or that N-methyl-N-methoxy-carbamic acid resulting from monolinuron and linuron transformation cannot be used for its growth.

Having isolates transforming DIU to 3,4-DCA we have been intensively searching for 3,4-DCA degraders. This led to the isolation of Achromobacter sp. SP1 able to use 3,4-DCA as sole carbon and nitrogen sources solely from the SED enrichment culture. Environmental survey of the quality of this river regularly shows the presence of traces of 3,4-DCA ( Pesce et al., 2010a) suggesting that microbial community of the sediments have a chronic history of exposure to this pollutant thereby explaining their adaptation to its degradation. One could hypothesize a low exposure of soil microbial community to 3,4-DCA in reason of its adsorption to soil thereby explaining why no 3,4-DCA degraders can be isolated from soil. SP1 was not only able to degrade 3,4-DCA but also a wide range of related anilines such as 3-chloro-4-methyl-aniline, 4-isopropylaniline, 4-chloroaniline, 3chloroaniline or 4-bromoaniline. This wide range catabolic ability has also been observed for Variovorax sp. WDL1 a linuron mineralizing strain which degrades 3,4-DCA, 3- and 4-chloroaniline and aniline ( Dejonghe et al., 2003). It is opposite to the specificity of Sphingomonas sp. SH an isoproturon mineralizing isolate only able to degrade 4- isopropylaniline (4-IA) ( Hussain et al., 2011). Interestingly we showed that SP1 had dcaQ gene which was $99 \%$ similar to that of C. testosteroni WDL7. This gene belonging to the dcaQTA1A2BR gene cluster encodes an enzyme responsible for the transformation of 3,4-DCA to chlorocatechol in Variovorax sp. Strain SRS16 ( Bers et al., 2011). This suggests that SP1 catalyses the degradation of anilines to chlorocatechol which might then be metabolized through the ortho-cleavage pathway ( Bers et al., 2011).

Our study suggests that the DIU-mineralization observed in the BS and SED enrichment cultures may result from the cooperation between two microbial populations one harbouring the puhA gene catalysing the upper pathway transforming DIU to 3,4-DCA and another one harbouring the dcaQ gene catalysing the lower pathway leading to the degradation of 3,4-DCA. This is an example of patchwork assembly of catabolic gene modules in different microbial populations (Arthrobacter sp. BS1, BS2 or SED1 [puhA]; Achromobacter sp. SP1 [dcaQ]) in order to use phenylurea herbicide as a source of carbon and energy. On different chemicals such as s-triazines, similar examples of cooperative metabolism were shown between populations catalyzing the upper pathway transforming atrazine to cyanuric acid and populations metabolising the lower pathway opening the s-triazine ring to completely degrade cyanuric acid ( Smith et al., 2005, Martin-Laurent et al., 2006, Udikovic-Kolic et al., 2010 and Udikovic-Kolic et al., 2012). The artificial consortium made of BS2 and SP1 led to the mineralization of DIU offering interesting perspectives to develop mixed inoculants for the bioaugmentation of buffer strip to improve their mitigation ability.

\section{Conclusions}

The isolation of different isolates able to degrade DIU confirmed that both terrestrial and aquatic microbial communities adapted in a similar manner to accelerated biodegradation of DIU. Interestingly, these isolates are closely related to A. globiformis strain D47 another DIU degrader isolated one decade ago. In addition, the DIU-degrading genetic potential was shown to be extremely conserved suggesting its widespread dispersion. Achromobacter sp. SP1 was shown to degrade 3,4- 
DCA formed during DIU degradation as well as a range of anilines. Altogether this study suggests that DIU mineralization is occurring through a cooperative metabolism between microbial populations catalysing the transformation of DIU to 3,4-DCA and others degrading this intermediary metabolite.

\section{Acknowledgments}

This project was supported by the French National Office for the Aquatic Environment (ONEMA). The authors thank Céline Guillemain of Irstea's Water Chemistry Laboratory (LAMA) for analysis of DIU.

\section{References}

Bers, K., Leroy, B., Breugelmans, P., Albers, P., Lavigne, R., Sorensen, S.R., Aamand, J., De Mot, R., Wattiez, R., Springael, D., 2011. A novel hydrolase identified by genomic-proteomic analysis of phenylurea herbicide mineralization by Variovorax sp strain SRS16. Appl. Environ. Microbiol. 77, 8754-8764.

Bonnet, J.L., Bonnemoy, F., Dusser, M., Bohatier, J., 2007. Assessment of the potential toxicity of herbicides and their degradation products to nontarget cells using two microorganisms, the bacteria Vibrio fischeri and the ciliate Tetrahymena pyriformis. Environ. Toxicol. 22, 78-91.

Claver, A., Ormad, P., Rodriguez, L., Ovelleiro, J.L., 2006. Study of the presence of pesticides in surface waters in the Ebro river basin (Spain). Chemosphere 64, 1437-1443.

Cullington, J.E., Walker, A., 1999. Rapid biodegradation of diuron and other phenylurea herbicides by a soil bacterium. Soil Biol. Biochem. 31, 677-686.

Dejonghe, W., Berteloot, E., Goris, J., Boon, N., Crul, K., Maertens, S., Hofte, M., De Vos, P., Verstraete, W., Top, E.M., 2003. Synergistic degradation of linuron by a bacterial consortium and isolation of a single linuron-degrading Variovorax strain. Appl. Environ. Microbiol. 69, 1532-1541.

Dellamatrice, P.M., Monteiro, R.T.R., 2004. Isolation of diuron-degrading bacteria from treated soil. Braz. Arch. Biol. Technol. 47, 999-1003.

Devers, M., Henry, S., Hartmann, A., Martin-Laurent, F., 2005. Horizontal gene transfer of atrazinedegrading genes (atz) from Agrobacterium tumefaciens St96- 4 pADP1::Tn5 to bacteria of maizecultivated soil. Pest Manage. Sci. 61, 870-880.

Ellegaard-Jensen, L., Knudsen, B.E., Johansen, A., Albers, C.N., Aamand, J., Rosendahl, S., 2014. Fungal-bacterial consortia increase diuron degradation in waterunsaturated systems. Sci. Total Environ. 466, 699-705.

Giacomazzi, S., Cochet, N., 2004. Environmental impact of diuron transformation: a review. Chemosphere 56, 1021-1032.

Gompertz, B., 1825. On the nature and function expressive of the law of human mortality, and on a new mode of determining the value of life contingencies. Phil. Trans. R. Soc. Lond. 115, 513-585.

Gürtler, V., Stanisich, V., 1996. New approaches to typing and identification of bacteria using the 16S-23S rDNA spacer region. Microbiology 142, 3-16.

Hongsawat, P., Vangnai, A.S., 2011. Biodegradation pathways of chloroanilines by Acinetobacter baylyi strain GFJ2. J. Hazard. Mater. 186, 1300-1307. 
Hussain, S., Devers-Lamrani, M., El Azhari, N., Martin-Laurent, F., 2011. Isolation and characterization of an isoproturon mineralizing Sphingomonas sp. strain SH from a French agricultural soil. Biodegradation 22, 637-650.

Khurana, J.L., Jackson, C.J., Scott, C., Pandey, G., Horne, I., Russell, R.J., Herlt, A., Easton, C.J., Oakeshott, J.G., 2009. Characterization of the phenylurea hydrolases A and B: founding members of a novel amidohydrolase subgroup. Biochem. J. 418, 431-441.

Martin-Laurent, F., Barres, B., Wagschal, I., Piutti, S., Devers, M., Soulas, G., Philippot, L., 2006. Impact of the maize rhizosphere on the genetic structure, the diversity and the atrazine-degrading gene composition of the cultivable atrazine-degrading communities. Plant Soil 282, 99-115.

Moncada, A., 2005. Environmental Fate of Diuron. Environmental Monitoring Branch. Department of Pesticide Regulation (<http://www.cdpr.ca.gov/docs/ empm/pubs/fatememo/diuron.pdf>).

Morin, S., Bottin, M., Mazzella, N., Macary, F., Delmas, F., Winterton, P., Coste, M., 2009. Linking diatom community structure to pesticide input as evaluated through a spatial contamination potential (Phytopixal): a case study in the Neste river system (South-West France). Aquat. Toxicol. 94, 28-39.

Pesce, S., Martin-Laurent, F., Rouard, N., Montuelle, B., 2009. Potential for microbial diuron mineralisation in a small wine-growing watershed: from treated plots to lotic receiver hydrosystem. Pest Manage. Sci. 65, 651-657.

Pesce, S., Lissalde, S., Lavieille, D., Margoum, C., Mazzella, N., Roubeix, V., Montuelle, B., 2010a. Evaluation of single and joint toxic effects of diuron and its main metabolites on natural phototrophic biofilms using a pollution-induced community tolerance (PICT) approach. Aquat. Toxicol. 99, 492499.

Pesce, S., Martin-Laurent, F., Rouard, N., Robin, A., Montuelle, B., 2010b. Evidence for adaptation of riverine sediment microbial communities to diuron mineralization: incidence of runoff and soil erosion. J. Soil. Sediment 10, 698-707.

Pesce, S., Bouchez, A., Montuelle, B., 2011. Effects of organic herbicides on phototrophic microbial communities in freshwater ecosystems. Rev. Environ.Contam. T. 214, 87-124.

Pesce, S., Beguet, J., Rouard, N., Devers-Lamrani, M., Martin-Laurent, F., 2013. Response of a diuron-degrading community to diuron exposure assessed by real-time quantitative PCR monitoring of phenylurea hydrolase A and B encoding genes. Appl. Microbiol. Biotechnol. 97, 1661-1668.

Schuler, L.J., Rand, G.M., 2008. Aquatic risk assessment of herbicides in freshwater ecosystems of south Florida. Arch. Environ. Contam. Toxicol. 54, 571-583.

Sharma, P., Suri, C.R., 2011. Biotransformation and biomonitoring of phenylurea herbicide diuron. Bioresour. Technol. 102, 3119-3125.

Smith, D., Alvey, S., Crowley, D.E., 2005. Cooperative catabolic pathways within an atrazinedegrading enrichment culture isolated from soil. FEMS Microbiol. Ecol. 53, 265-273.

Sorensen, S.R., Aamand, J., 2003. Rapid mineralisation of the herbicide isoproturon in soil from a previously treated Danish agricultural field. Pest Manage. Sci. 59, 1118-1124.

Sorensen, S.R., Ronen, Z., Aamand, J., 2001. Isolation from agricultural soil and characterization of a Sphingomonas sp. able to mineralize the phenylurea herbicide isoproturon. Appl. Environ. Microbiol. $67,5403-5409$.

Sorensen, S.R., Albers, C.N., Aamand, J., 2008. Rapid mineralization of the phenylurea herbicide diuron by Variovorax sp. strain SRS16 in pure culture and within a two-member consortium. Appl. Environ. Microbiol. 74, 2332-2340. 
Stork, P.R., Bennett, F.R., Bell, M.J., 2008. The environmental fate of diuron under a conventional production regime in a sugarcane farm during the plant cane phase. Pest Manage. Sci. 64, 954-963.

Sun, J.Q., Huang, X., Chen, Q.L., Liang, B., Qiu, J.G., Ali, S.W., Li, S.P., 2009. Isolation and characterization of three Sphingobium sp. strains capable of degrading isoproturon and cloning of the catechol 1,2-dioxygenase gene from these strains. World J. Microbiol. Biotechnol. 25, 259-268.

Tadonleke, R.D., LeBerre, B., Perreau, F., Humbert, J.F., 2009. Responses of lake bacterioplankton activities and composition to the herbicide diuron. Aquat. Toxicol. 94, 103-113.

Thompson, J.D., Gibson, T.J., Plewniak, F., Jeanmougin, F., Higgins, D.G., 1997. The ClustalX Windows interface: flexible strategies for multiple sequence alignment aided by quality analysis tools. Nucl. Acids Res. 24, 4876-4882.

Travkin, V.M., Solyanikova, I.P., Rietjens, I., Vervoort, J., van Berkel, W.J.H., Golovleva, L.A., 2003. Degradation of 3,4-dichloro- and 3,4-difluoroaniline by Pseudomonas fluorescens 26-K. J. Environ. Sci. Heal. B 38, 121-132.

Turnbull, G.A., Ousley, M., Walker, A., Shaw, E., Morgan, J.A.W., 2001. Degradation of substituted phenylurea herbicides by Arthrobacter globiformis strain D47 and characterization of a plasmidassociated hydrolase gene, puhA. Appl. Environ. Microbiol. 67, 2270-2275.

Udikovic-Kolic, N., Hrsak, D., Devers, M., Klepac-Ceraj, V., Petric, I., Martin-Laurent, F., 2010. Taxonomic and functional diversity of atrazine-degrading bacterial communities enriched from agrochemical factory soil. J. Appl. Microbiol. 109, 355-367.

Udikovic-Kolic, N., Scott, C., Martin-Laurent, F., 2012. Evolution of atrazine-degrading capabilities in the environment. Appl. Microbiol. Biotechnol. 96, 1175-1189.

\section{Supplementary data}

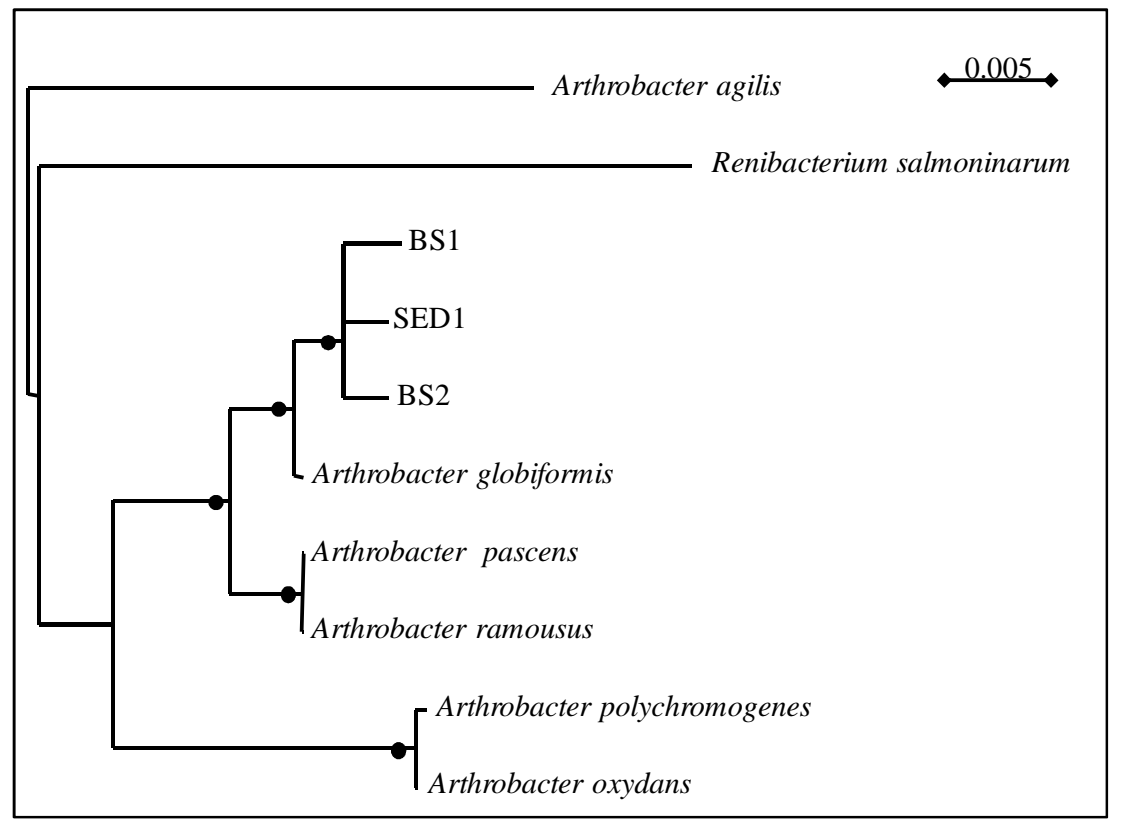

Phylogenetic tree was established with 16S rRNA sequences used in the paper of Turnbull et al. 2001 describing the isolation and the characterization of Arthrobacter sp. D47 from Deep Slade soil (UK). Only branches having a bootstrap values up to 900 of 1000 iterations are highlighted by a black dot. The strains used for phylogenetic analysis and the GenBank accession numbers of the 16S rRNA genes are: Arthrobacter agilis (X80748), Renibacterium salmoninarum (X51601), Arthrobacter globiformis (X80736), Arthrobacter ramousus (X80742), Arthrobacter pascens (X80740), Arthrobacter oxydans (X83408) and Arthrobacter polychromogenes (X80741). 